\title{
Legionella pneumophila pangenome reveals strain-specific virulence factors
}

\author{
Giuseppe D’Auria ${ }^{1,2^{*}}$, Nuria Jiménez-Hernández ${ }^{1,2}$, Francesc Peris-Bondia ${ }^{3}$, Andrés Moya ${ }^{1,2,3}$, Amparo Latorre ${ }^{1,2,3}$
}

\begin{abstract}
Background: Legionella pneumophila subsp. pneumophila is a gram-negative $\gamma$-Proteobacterium and the causative agent of Legionnaires' disease, a form of epidemic pneumonia. It has a water-related life cycle. In industrialized cities L. pneumophila is commonly encountered in refrigeration towers and water pipes. Infection is always via infected aerosols to humans. Although many efforts have been made to eradicate Legionella from buildings, it still contaminates the water systems. The town of Alcoy (Valencian Region, Spain) has had recurrent outbreaks since 1999. The strain "Alcoy 2300/99" is a particularly persistent and recurrent strain that was isolated during one of the most significant outbreaks between the years 1999-2000.
\end{abstract}

Results: We have sequenced the genome of the particularly persistent L. pneumophila strain Alcoy 2300/99 and have compared it with four previously sequenced strains known as Philadelphia (USA), Lens (France), Paris (France) and Corby (England).

Pangenome analysis facilitated the identification of strain-specific features, as well as some that are shared by two or more strains. We identified: (1) three islands related to anti-drug resistance systems; (2) a system for transport and secretion of heavy metals; (3) three systems related to DNA transfer; (4) two CRISPR (Clustered Regularly Interspaced Short Palindromic Repeats) systems, known to provide resistance against phage infections, one similar in the Lens and Alcoy strains, and another specific to the Paris strain; and (5) seven islands of phage-related proteins, five of which seem to be strain-specific and two shared.

Conclusions: The dispensable genome disclosed by the pangenomic analysis seems to be a reservoir of new traits that have mainly been acquired by horizontal gene transfer and could confer evolutionary advantages over strains lacking them.

\section{Background}

Legionella pneumophila is a gram-negative facultative intracellular pathogen, identified as the infectious agent of the Legionnaire's disease (LD) or Legionellosis in 1977 [1]. It is found in aquatic environments parasitizing its natural hosts, amoebae and protozoa. From this environment, Legionella can colonize water treatment plants, such as refrigeration towers, potable water pipes, etc., and can cause infections in humans, when infected aerosols are inhaled [2,3]. Despite efforts to keep water systems free of Legionella, this pathogen is still causing infection throughout the world, including Spain, where it is endemic in some areas. From 1989 to 2005, around 310 outbreaks with 2,974 cases were recorded worldwide. In 2002 and 2005 there were two important

\footnotetext{
* Correspondence: dauria_giu@gva.es

${ }^{1}$ CIBER en Epidemiología y Salud Pública (CIBERESP), Barcelona, Spain
}

epidemic events with 1,461 and 1,292 cases respectively. In Alcoy, an industrial town in the Valencian Region (Spain), a large outbreak occurred during 1999-2000. A strain that had caused several outbreaks and many cases, named "Alcoy 2300/99", was isolated from a patient in that outbreak [4]. Since then, recurrent epidemics in Alcoy have harbored Alcoy 2300/99.

Currently, the genomes of five L. pneumophila strains are available: Philadelphia (Lpg, USA) [5], Lens (Lpl, France) and Paris (Lpp, France) [6], Corby (Lpc, England) [7] and Alcoy (Lpa, Spain) (reported in this work). As with the majority of other pathogenic Legionella strains, immunoassay analysis defined them as belonging to the serogroup 1 [8]. A phylogeny based on Multi Locus Sequence Typing (MLST) showed that all strains are closely related, Alcoy and Corby being the closest [9]. 
Several features relating to the virulence of L. pneumophila are well known. For example, the mechanisms responsible for entry into the macrophages $[10,11]$, the intracellular (host) trafficking of effectors [12] and the membrane-associated protein involved in virulence $[9,13]$. The data available disclose an almost complete physiology of this organism and its relationships with protozoa and human macrophages. An interesting question relating to L. pneumophila is its high rate of DNA exchange, not only within species and other closelyrelated bacteria, but also with eukaryotic organisms [14]. Comparative genomics can give clues about the extent of this process. Nowadays, the genome sequencing of strains belonging to the same species offers the possibility of defining their pangenome, which helps in understanding the evolutionary dynamics of microbial species. The pangenome comprises the core-genome, made up of the genes shared by all strains, and the accessory or dispensable genome compartment, consisting of the genes that are strain-specific or shared by only some of the strains [15]. Pangenome studies can disclose characteristics that are not easily perceptible using standard annotation analysis [16]. For example, pangenome studies have facilitated identification of virulence factors or anti-drugs systems in Escherichia coli and Streptococcus agalactiae $[17,18]$. The dispensable genome compartment can provide evidence of lateral gene transfer events that have occurred during the evolutionary history of a strain, probably offering additional evolutionary potential to the organism.

In this work, we report the main genomic features of L. pneumophila strain Alcoy 2300/99 and compare it with the four previously sequenced strains. A detailed description of the L. pneumophila pangenome is provided, and strain-specific features are catalogued in terms of "islands". Several islands containing virulence factors were identified and, where possible, their evolutionary origins were also hypothesized. Although the strains are phylogenetically closely related, the pangenomic approach allowed identification of distinctive features, such as anti-drug related islands, strain-specific transport or secretion systems, DNA transfer-related islands, CRISPR (Clustered Regularly Interspaced Short Palindromic Repeats) systems, and integrated phage insertions.

\section{Results and Discussion}

\section{General features of the L. pneumophila Alcoy (Lpa)}

\section{genome}

A total of 36,974 and 215,350 sequences were obtained by the Sanger method (with an average length of $781 \mathrm{nt}$ ) and 454-technology (with an average length of $242 \mathrm{nt}$ ), respectively. The contigs were finally assembled in one continuous strand, with average consensus coverage of $22.1 \times$. The Lpa chromosome length was of $3,516,335$ base pairs, and 3,197 open reading frames were identified. The GC content was $38 \%$ and 1,175 predicted proteins were found to have unknown functions, representing $36.7 \%$ of all CDSs. Similar to Lpc and Lpg genomes, no plasmids were found in Lpa. Table 1 summarizes the main features of this genome compared to the other four L. pneumophila sequenced strains.

\section{Pangenome of L. pneumophila}

Figure 1 summarizes the results obtained from comparing the five complete genomes of pathogenic L. pneumophila strains in relation to the orthologs/accessory gene distribution. The pangenome consists of 2,957 CDS with a core of 1,979 genes (66.9\%) and a dispensable genome of 978 CDSs (33.1\%). A total of 342 genes were found to be specific to the Lpa (53), Lpc (48), Lpg (88), Lpl (64) and Lpp (98) genomes. It is worth mentioning that 287 out of these 342 accessory genes (83.9\%) were hypothetical proteins.

Lpa and Lpc are the strains that share most genes; 2,560 out of 3,196 in Lpa (80\%) and 3,207 in Lpc $(79.8 \%)$. This result is in agreement with the phylogenetic tree obtained using MLST [9]. Compared with the remaining genomes, Lpa and Lpc share 2,208 (69.1\%) and 2,181 (68\%) genes with Lpl; 2,271 (71.1\%) and 2,284

Table 1 Main features of $L$. pneumophila genomes

\begin{tabular}{|c|c|c|c|c|c|}
\hline Features & $\begin{array}{l}\text { Alcoy } \\
\text { (Lpa) }\end{array}$ & $\begin{array}{l}\text { Corby } \\
\text { (Lpc) }\end{array}$ & Philadelphia (Lpg) & $\begin{array}{l}\text { Paris } \\
\text { (Lpp) }\end{array}$ & $\begin{array}{l}\text { Lens } \\
\text { (Lpl) }\end{array}$ \\
\hline Accession Number & CP001828 & NC_009494 & NC_002942 & NC_006368 & NC_006369 \\
\hline Serotype & 1 & 1 & 1 & I & I \\
\hline Genome length (bp) & $3,516,335$ & $3,576,470$ & $3,397,754$ & $3,503,610$ & $3,345,687$ \\
\hline Plasmid & 0 & 0 & 0 & 1 & 1 \\
\hline GC content (\%) & 38.38 & 38.48 & 38.27 & 38.37 & 38.42 \\
\hline Coding genes (\%) & 86 & 86 & 88 & 87 & 86 \\
\hline Ribosomal operons & 3 & 3 & 3 & 3 & 3 \\
\hline Islands bp (\%) & $157,442(4.48)$ & $217,089(6.07)$ & $180,555(6.13)$ & $163,637(4.67)$ & $180,986(5.40)$ \\
\hline Average GC of islands (\%) & 37.75 & 37.43 & 36.01 & 38.17 & 37.66 \\
\hline
\end{tabular}




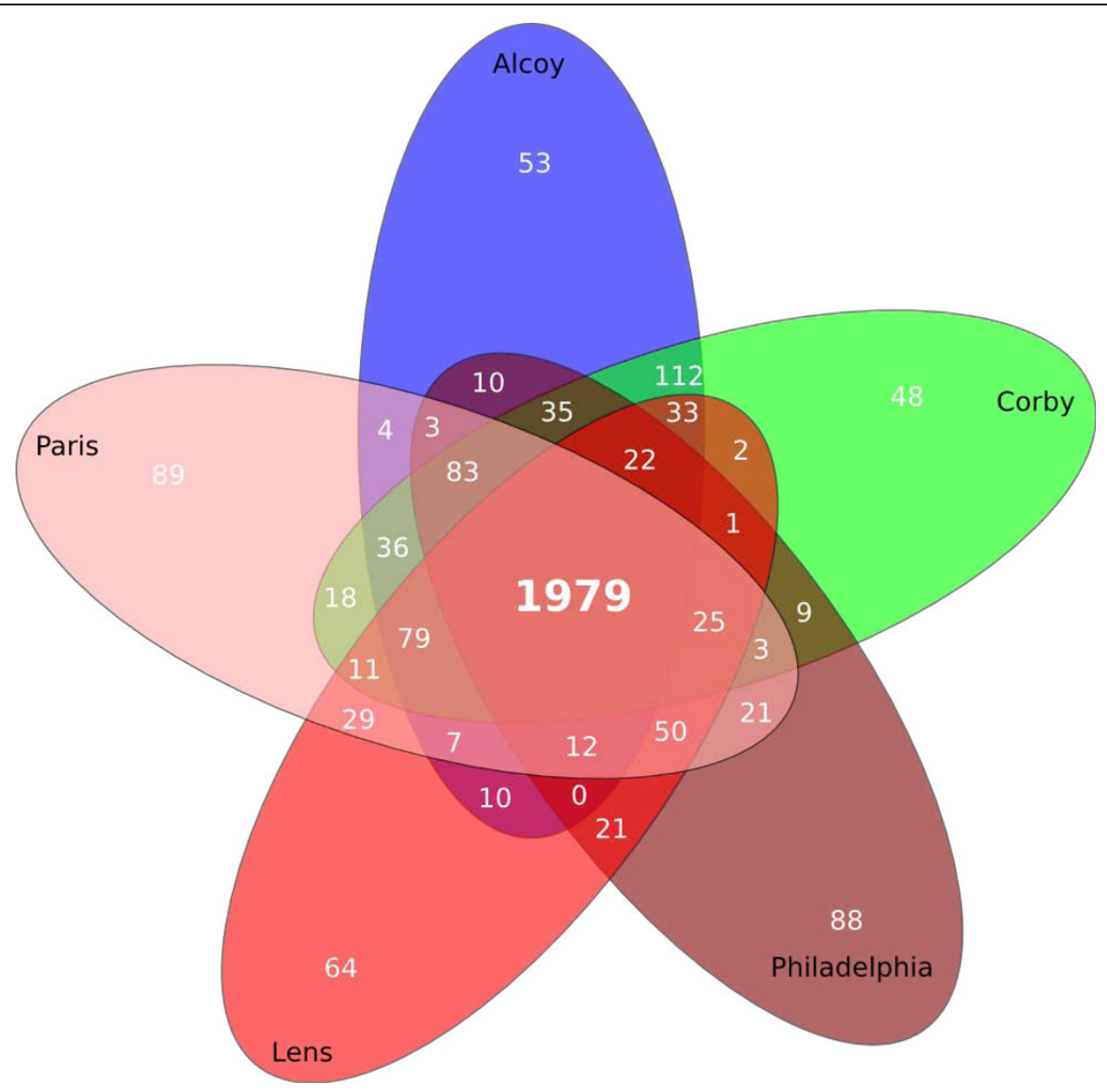

Figure 1 Pangenome overview. The Venn diagram showing the core genome and the genes specific of the strains L. pneumophila Alcoy, Philadelphia, Lens, Paris and Corby. Genes overlapping at least $70 \%$ length and $80 \%$ of similarity were considered orthologs.

(71.2\%) with Lpp; and 1,802 (56.4\%) and 1,776 (55.4\%) with Lpg, respectively. Similarly, Lpp and Lpl also seem to be close-related with a shared genome of 2,207 genes out of 2,877 for Lpl (76.7\%) and 3,026 for Lpp (73\%). Finally, Lpg seems to be the most distantly related, sharing 2,207 genes with $\mathrm{Lpl}(75 \%)$ and 1,792 (60.1\%) with Lpp.

Figure 2 shows the application of the rarefaction methodology on the gene clusters from multiple genomes belonging to the same species. L. pneumophila tends to reach a plateau, although, according to Tettelin and collaborators [18], it should be considered an open pangenome similarly to what happens in other pathogenic organisms, such as the pangenomes from the same species of Streptococcus agalactiae, S. pyogenes and Staphylococcus aureus [18,19]. In the case of E. coli, despite the growing number of complete genomes, its pangenome is still far from fully described [17]. It has also been reported that clinically related pathogenic bacteria posses a lower level of variation than free-living bacteria, which is probably due to niche restriction that could lead to a wider core genome $[20,21]$.

\section{Functional classification of core and dispensable genes}

Genes belonging to the core and dispensable genomes have been classified according to their predicted function based on COG categories (Figure 3). L. pneumophila is characterized by quite a high number of hypothetical proteins, for which annotation is still incomplete. Of the 1,979 genes belonging to the core genome, 1,131 (57\%) were attributed to a COG category ( $e$ value less than $10^{-15}$ ), and in the case of dispensable genome, only 179 out of 978 (18.2\%). These results are in agreement with those obtained in other studies, where hypothetical genes, and even genes with unknown function are, in the majority, in the dispensable genome [18]. Although the major proportion of the CDSs for which a function could be predicted (according to the COG database) falls within the core genome, minor differences between the two compartments were observed 


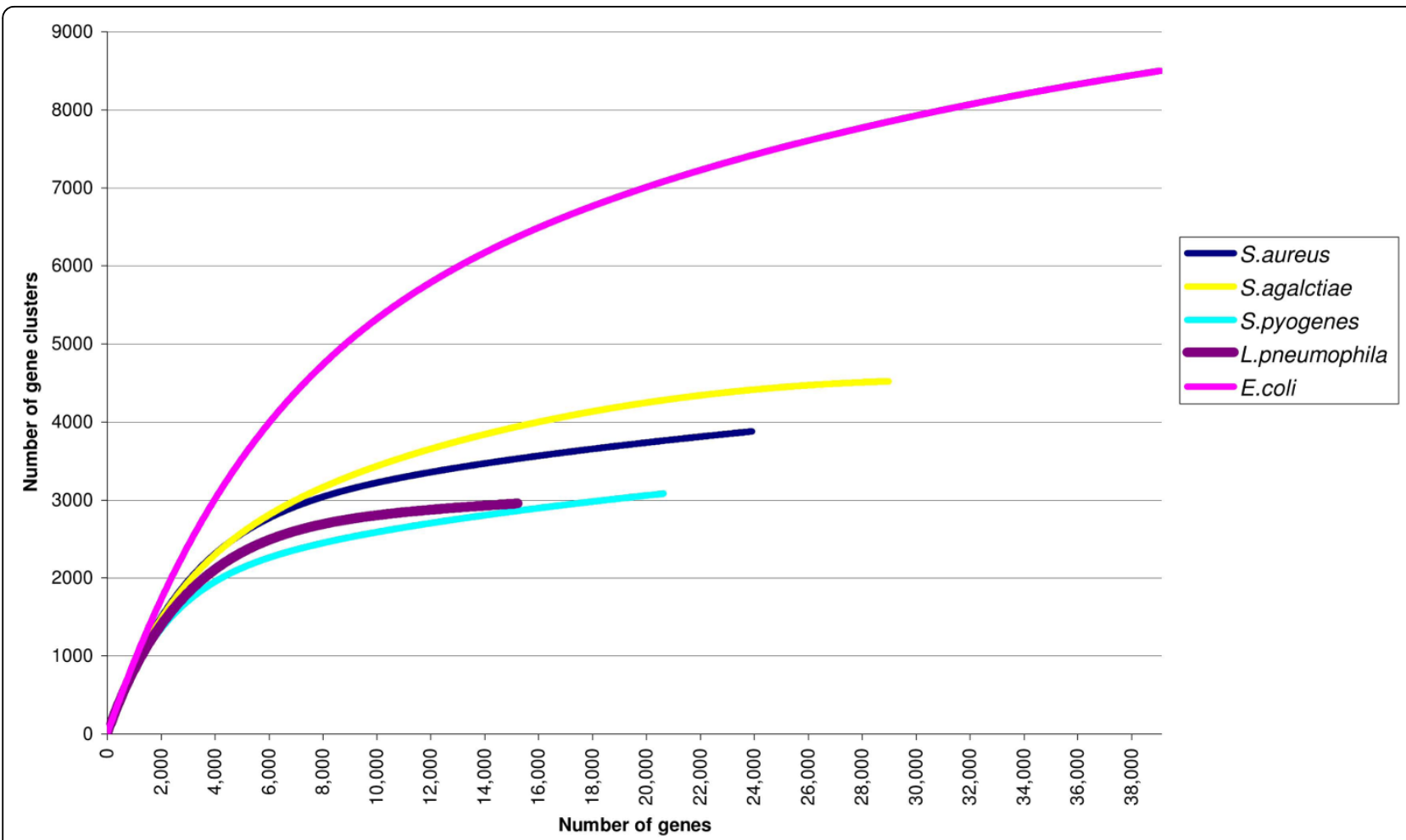

Figure 2 Pangenome state. Rarefaction curves applied to different strains of L. pneumophila (5 genomes), E. coli (8 genomes), Streptococcus pyogenes (8 genomes), S. agalactiae (8 genomes), and Staphilococcus aureus (9 genomes). See Additional file 6 for accession numbers of used genomes.

for defense mechanisms (V) and intracellular trafficking, secretion and vesicular transport $(\mathrm{U})$ categories.

\section{Genomic islands}

Although the five strains are highly syntenic, most of the genes that do not belong to the core genome are part of genomic islands, absent in at least one of the genomes. Table 2 reports the islands identified for each strain, Figure 4 describes the island positions for each genome following Table 2 classifications, while Figure 5a shows the hypothesis of islands histories according to the MLST tree topology obtained by D'Auria et al. [9]. Figure 5b shows the alignment of the five genomes, the locally collinear blocks and the position of the islands according to their genome locations. Twenty-eight islands were identified belonging to six different types (see Additional File 1). Only one island (R1, see below) is present in all five genomes; eighteen are strain-specific, probably acquired by horizontal gene transfer (HGT) events; five are common to Lpa/Lpc genomes (probably acquired by the Lpa/Lpc common ancestor), whereas one island (DT3, see below) could be interpreted as having been lost in the common ancestor of Lpa/Lpc genomes.

\section{Resistance-related islands ( $R$ )}

We have identified two types of resistance-related islands, R1 and R2 (see Figure 4 and Figure 5, blue track). $\mathrm{R} 1$ maintains the same position, around $60 \mathrm{~Kb}$ at the beginning of the chromosome, in the five strains, inserted in a tRNA ${ }^{\text {asn }}$ site, although the organization and content is different. In Lpa, Lpc and Lpg, the island is similar and contains several hypothetical proteins as well as a methylase (lpa00094, LPC_0075, lpg0060), followed by a multi-drug resistance protein (lpa00095, LPC_0076, lpg0061). In the Paris strain, the island also begins with a methylase (lpp0063) and in the Lens strain with a putative transposase (lpl0064). However, although the position of the gene coding for the methylase is quite similar in both genomes, the alignment is different, due to a relatively big deletion in $\mathrm{Lpl}$, whereas in Lpp it is followed by the antibiotic persistence-related system HipB/HipA (lpp0065, lpp0064) with no homolog in other Legionella strains. Although the mechanism of this system is still not well known, much evidence points to HipA as a toxin with bacteriostatic activity which binds DNA/RNA, blocking macromolecule synthesis until HipB binds HipA, releasing the DNA/RNA so microbial cells can survive extended exposure to drugs $[22,23]$. This island is followed by various phage-related proteins, elements of the Xre family of transcriptional regulators, an LvrA protein, and three transporters (lpp0077, lpp0078, lpp0079) of which the lpp0077 is similar to the acriflavine multi-drug efflux pump [24]. 


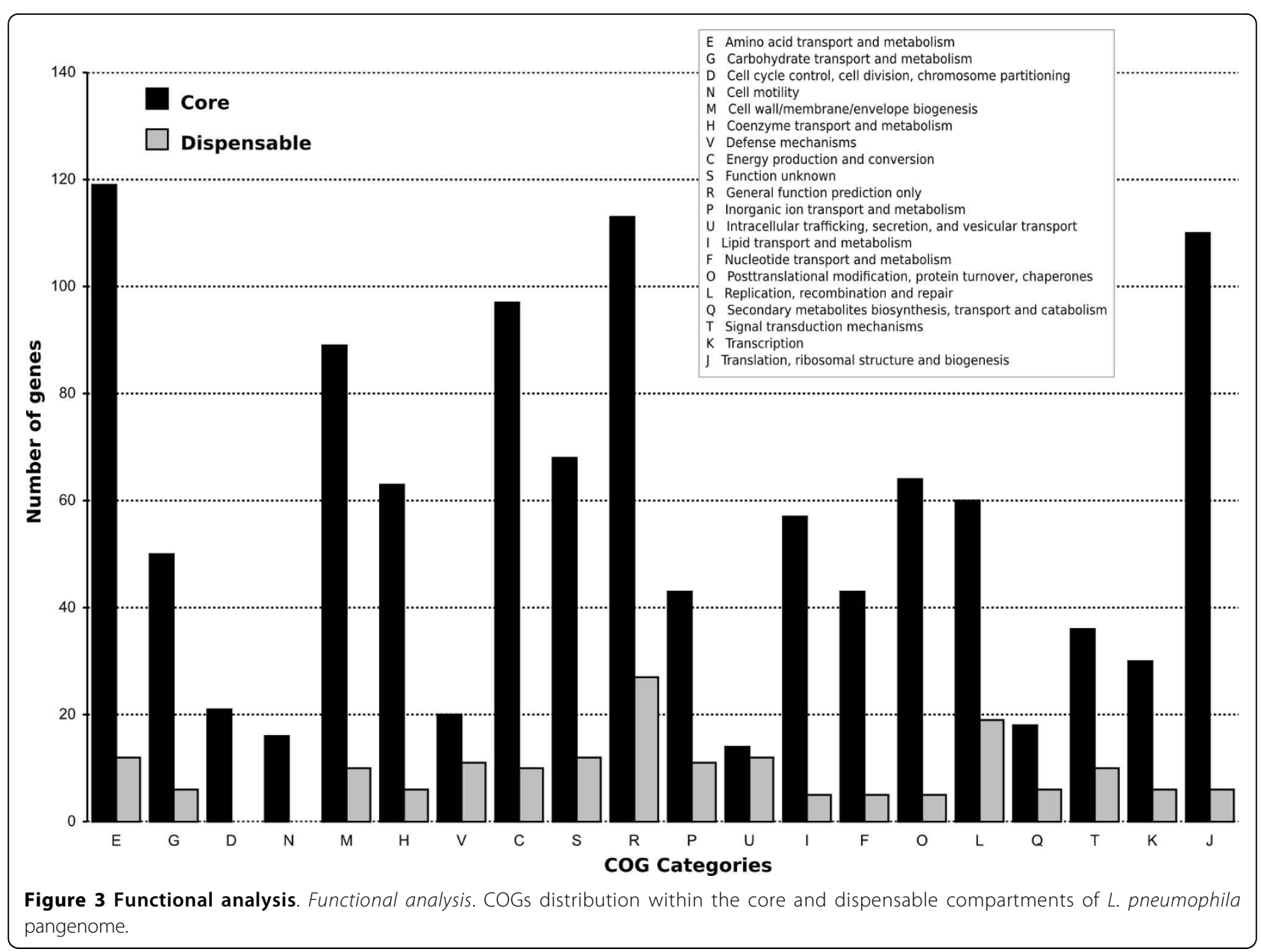

Finally, the island ends with three hypothetical proteins and an IS4-family transposase (lpp0083). Several genes such as the TraK, the LvrA-related protein and the phage-related integrases are also maintained in the same positions in the Alcoy, Corby, Lens and Philadelphia strains. A similar system (R2), was also found in the Lens strain. It is a small region containing transposases as well as two homologs of a stability system $S t b D E$ (lpl1587, lpl1588). This island was originally associated with plasmids [25], but it has also been found on the chromosomes of other pathogenic bacteria [26].

\section{Transport/secretion systems (TS)}

Only one TS island has been found in the Lpa and Lpc genomes. TS1 in the Alcoy strain is composed of 16 ORFs (lpa01590 to lpa01614). Three of these ORFs, lpa01601, lpa01599 and lpa01598, are related to the cobalt/zinc/cadmium efflux HelABC transport system that provides resistance against these heavy metals [27]. They are followed by lpa01604, which codes for the metallo-regulator ArsR that, in the presence of metals, derepresses the operator/promotor DNA, thereby activating the transcription of downstream genes [28]. As happens in other islands, this ends with a phage integrase and three transposase-related ORFs, indicating a possible exogenous origin in Legionella. It is worth mentioning that all five genomes carry a $\mathrm{Hel} \mathrm{ABC}$ operon belonging to the core genome, while Alcoy and Corby strains also possess the two additional above-mentioned systems.

In the Corby strain, TS1 is bigger than in the Alcoy, spanning about $50 \mathrm{~kb}$. It contains the previously mentioned HelABC operon (LPC_1847- LPC_1849), in addition to the HelABC systems present as part of the core genome (LPC_02269, LPC_02270, LPC_02271), a transposase (LPC_1856) and a phage repressor protein (LPC_1857). The first 9 ORFs of the TS1c island (mainly hypothetical proteins and one transposase) are syntenic with the Paris ND9 island (see below). The island continues for about $19 \mathrm{~kb}$ with apparently no synteny with other genomes and then regains synteny with the Paris ND10 island.

Interestingly, the TS1a-HelABC genes seem to be more similar to the core Lpl operon than to that of Lpc TS1 while TS1c-HelABC genes are more similar to 
Table 2 Accessory genes islands

\begin{tabular}{|c|c|c|c|c|c|c|c|}
\hline $\begin{array}{l}\text { Island } \\
\text { type }\end{array}$ & Genome & Details & Position & $\begin{array}{l}\text { \# of } \\
\text { CDSs }\end{array}$ & Length & Transposase & $\begin{array}{c}\mathrm{GC} \\
\%\end{array}$ \\
\hline \multicolumn{8}{|c|}{ Resistance related islands $(R)$} \\
\hline & Alcoy & Contains several multi-drug related protein & $68252-87883$ & 22 & 19632 & + & 3534 \\
\hline & Corby & & $69153-88336$ & 22 & 19184 & + & 35.30 \\
\hline & Philadelphia & & $68096-77398$ & 9 & 9303 & + & 33.96 \\
\hline \multirow[t]{2}{*}{ R1 } & Paris & $\begin{array}{l}\text { Antibiotic persistance related system HipB/HipA; Phage related } \\
\text { proteins Xre, and multi-drug efflux pump }\end{array}$ & $66766-85124$ & 21 & 18359 & + & 38.05 \\
\hline & Lens & Methylase, prophage integrase, TraK homologous & $68898-83207$ & 10 & 14310 & + & 36.77 \\
\hline R2 & Lens & Stability system StbDE & $1755441-1767402$ & 10 & 11962 & + & 40.83 \\
\hline \multicolumn{8}{|c|}{ Transport/secretion systems(TS) } \\
\hline TS1 & Alcoy & $\begin{array}{l}\text { Cobalt/zinc/cadmium efflux transporter helABC; ArsR regulatory } \\
\text { proteins }\end{array}$ & $1251900-1268281$ & 16 & 16382 & + & 40.75 \\
\hline \multicolumn{7}{|c|}{ DNA transfer (DT) } & 39.40 \\
\hline \multirow{2}{*}{ DT1 } & Alcoy & $\begin{array}{l}\text { tra and trb conjugal transfer proteins, Rac integrase roteins, } \\
\text { htpX protease prophage regulatory protein alpA }\end{array}$ & $181578-230836$ & 61 & 49259 & - & 38.56 \\
\hline & Corby & & $181312-241161$ & 63 & 59850 & - & 39.11 \\
\hline \multirow{3}{*}{ DT2 } & Alcoy & $\begin{array}{l}\text { Putative RNA helicase, two putative restrictases, tra and trb } \\
\text { conjugal transfer proteins. }\end{array}$ & $609680-648761$ & 40 & 39082 & - & 39.52 \\
\hline & Corby & & $613277-656674$ & 42 & 43398 & - & 39.25 \\
\hline & Paris & Plasmid-like elements containing $/ v h, / v r$ & $183831-234043$ & 45 & 50213 & - & 39.90 \\
\hline \multirow[t]{2}{*}{ DT3 } & Philadelphia & & $1353613-1400796$ & 45 & 47184 & + & 37.47 \\
\hline & Lens & & $172914-239494$ & 64 & 66581 & - & 39.13 \\
\hline \multicolumn{8}{|c|}{ CRISPR systems (C) } \\
\hline \multirow{2}{*}{ C1 } & Lens & Really similar CRISPR system & $3226572-3248046$ & 16 & 21475 & - & 40.75 \\
\hline & Alcoy & & $1169086-1179252$ & 6 & 10167 & - & 38.49 \\
\hline $\mathrm{C} 2$ & Paris & *Part of above P2 island & .. & $* 45$ & $\ldots$ & $\ldots$. & $\ldots$ \\
\hline \multicolumn{8}{|c|}{ Integrated phage related (PR) } \\
\hline & Alcoy & MviN virulence factor & $1292822-1329842$ & 30 & 37021 & + & 36.14 \\
\hline \multirow[t]{2}{*}{ PR1 } & Corby & & $1278158-1324830$ & 49 & 46673 & + & 36.23 \\
\hline & Philadelphia & & $1167775-1185079$ & 15 & 17305 & + & 37.33 \\
\hline PR2 & Philadelphia & Mainly transposases & $173401-183804$ & 10 & 10404 & + & 35.14 \\
\hline \multirow{2}{*}{ PR3 } & Corby & Ankyrine containing domain & $2493848-2532367$ & 11 & 38520 & + & 38.11 \\
\hline & Alcoy & & $2486005-2509743$ & 6 & 23739 & + & 37.00 \\
\hline PR4 & Lens & Plasmid maintenance killer/antidote system & $1190582-1219661$ & 30 & 29080 & + & 34.79 \\
\hline PR5 & Alcoy & Bacilysin system & $2756698-2784130$ & 33 & 27433 & + & 36.78 \\
\hline PR6 & Philadelphia & Type IV secretion system & $2296937-2366483$ & 64 & 69547 & + & 38.04 \\
\hline PR7 & Paris & Probable phage integration & $2408503-2419758$ & 16 & 11256 & - & 36.62 \\
\hline \multicolumn{8}{|c|}{ Not well defined (ND) } \\
\hline ND1 & Paris & Cytochrome 0 & $325750-334842$ & 10 & 9093 & - & 38.77 \\
\hline ND2 & Paris & Carbon storage regulator & $1192799-1199183$ & 10 & 6385 & - & 36.76 \\
\hline ND3 & Paris & Nickel/Cobalt type II transport systems & $1353408-1356362$ & 6 & 2955 & - & 39.63 \\
\hline ND4 & Philadelphia & No clear role & $1439890-1450778$ & 10 & 10889 & + & 35.17 \\
\hline ND5 & Philadelphia & Mainly transposases and phages integrases & $2892417-2904871$ & 16 & 12455 & + & 34.97 \\
\hline ND6 & Corby & No clear role & $1182245-1189318$ & 9 & 7074 & - & 36.78 \\
\hline ND7 & Lens & Incomplete HipA/HipB system & $2605322-2617191$ & 12 & 11870 & - & 34.77 \\
\hline ND8 & Paris & No clear role & $1733202-1746135$ & 7 & 12934 & + & 38.09 \\
\hline ND9 & Paris & No clear role & $2654264-2661604$ & 9 & 7341 & + & 37.57 \\
\hline
\end{tabular}


Table 2: Accessory genes islands (Continued)

\begin{tabular}{|c|c|c|c|c|c|c|c|}
\hline ND10 & Paris & No clear role & $2725059-2776774$ & 61 & 51716 & + & 37 \\
\hline \multirow{2}{*}{ ND11 } & Alcoy & Mainly transposases & $3003085-3015471$ & 28 & 12387 & + & 37.15 \\
\hline & Corby & & $3443118-3451379$ & 14 & 8262 & + & 35.00 \\
\hline ND12 & Lens & No clear role & $2824095-2849378$ & 16 & 25284 & - & 36.77 \\
\hline ND13 & Corby & No clear role & $2833804-2842585$ & 10 & 8782 & + & 37.42 \\
\hline
\end{tabular}

First column describes the name of the island and the genome of provenance. Following columns report a short description, the position on the genomes, the number of CDSs contained in the island, the length, the presence $(+) /$ absence $(-)$ of transposase genes and the GC content.

those of the Lpg genome. Conversely, core HelABC genes in Lpa and Lpc are highly related.

\section{DNA transfer-related islands (DT)}

Three DT islands have been identified (DT1, DT2 and DT3). DT1 and DT2 correspond to the Trb-2 and Trb-1 islands described by Glöckner and collaborator for the Corby strain [7]. We have found that both islands are also present in the Alcoy strain, although DT2a is shorter than DT2c, thus suggesting that Lpa and Lpc acquired these systems via DNA transfer prior to their divergence (see Figure 5, green track). Some remarkable features of DT1 are: a phage repressor (lpa00219, LPC_0166), a set of tra and trb (conjugal transfer proteins) operons, a putative lamboid prophage Rac integrase (lpa00266, LPC_0199), another integrase (lpa00270, LPC_0202), an $h t p X$ protease (lpa00275, LPC_0205), and a prophage regulatory protein alpA (lpa00278, LPC_0208). In the DT2 island, both strains share a putative RNA helicase (lpa00835, LPC_2785), two putative restriction enzymes (LPC_2788, LPC_2790, lpa00832, and lpa_00829) and the set of the tra and trb operons. Glöckner and collaborators [7] described that Trb-1 (here DT2) is active and could be transferred to other Legionella. In both genomes, DT1 and DT2 are integrated, respectively, at the tmRNA and tRNA ${ }^{\text {pro }}$ sites.

Finally, we have identified a DNA transfer island, DT3, in all strains with the exception of the Corby and Alcoy ones. It is worth mentioning that this island was previously described in Lpg and Lpp, as an integrated plasmid-like element $[5,29]$. It contains lvh (Legionella vir homolog), a type IV secretion system involved in conjugation [30,31]. The lvr (Legionella vir region) is located downstream where LvrA is homologous to the CsrA repressor, important for the inhibition of post-exponential phase activity (such as DNA transfer) [32]. A CRISPR system was identified at the beginning of this island.
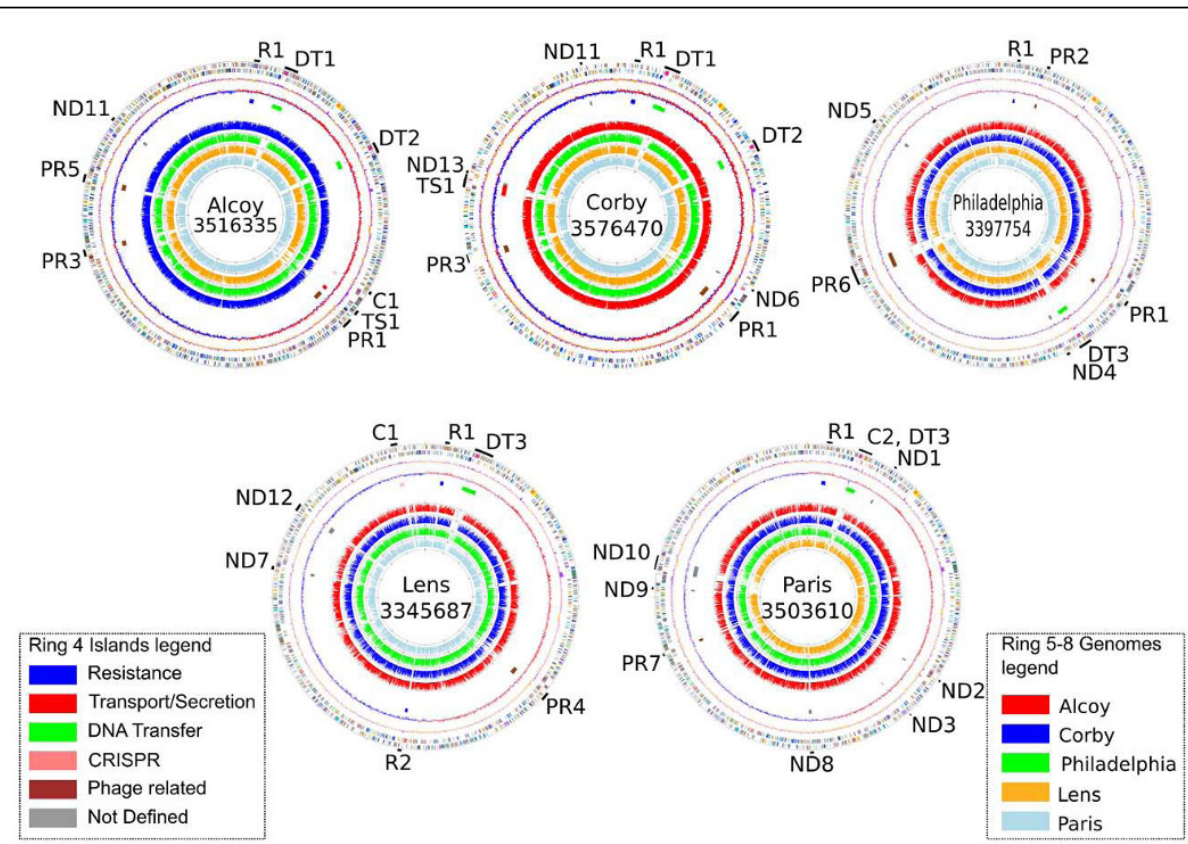

Figure 4 Strain related orthology maps. Strain related orthology maps. Each map shows comparative analysis based on each genome. From outside to inside: ring 1, gene positions according with COG categories color code; ring 2, GC content; ring 3, GC Skew; ring 4, island positions (color code according with islands legend); rings 5 to 8 show the BLAST homology for each gene versus the other four genomes (color code according with genomes legend). 


\section{Clustered Regularly Interspaced Short Palindromic Repeats (CRISPR) systems (C)}

CRISPR are bacteriophage resistance systems [33] that have been identified in the Alcoy and Lens (C1), and in Paris (C2) strains (Figure 5, pink track). Lpl also possesses one almost identical CRISPR system on the plasmid. Phylogenetic analyses reveal how Alcoy CAS (CRISPR-associated genes) genes are more closely related to the ones on the plasmidic CRISPR of the Lens strain than to the chromosomal one. The CAS genes identified were Cas1 (lpl2837, plpl0052, lpa01472), Cas3-helicase (plpl0051, lpl2838, lpa01473), and lpa01474 and lpa01475, which are two conserved proteins in bacteria, but with no relatives in other Legionella genomes. lpa01476, plpl0047 and lpl2842 belong to the CRISPR-associated Csy4 family proteins (see Additional file 2). Downstream of this cluster of proteins begins the repeats of the CRISPR system (see Additional file 3). The Alcoy and Lens strain repeats are almost identical, except for one base (adenine in the Alcoy strain and cytosine in the Lens, GTT(A/C)ACTGCCGCACAGGCAGCTTAGAA). Fifty-seven direct repeats were found in Lpa, while 53 in the Lpl plasmid, and two clusters of 52 and 12 on the Lpl chromosome. None of the Alcoy spacers were found to be similar to other spacers from the CRISPR database [34].

The Paris strain genome hosts a CRISPR system, located at the beginning of the DT3 island. The first
CRISPR-related protein is coded by lpp0160 and shows a weak similarity to a putative CRISPR-associated large protein. The cluster is followed by Cas1 (lpp0161), Cas2 (lpp0162), Cas4 (lpp0163) and 34 direct repeats. Interestingly, the repeats and the CRISPR-associated genes are not related to the ones found in the Lpa and Lpl genomes.

\section{Phage related islands (PR)}

Up to seven different phage-related islands have been found. The Alcoy and Corby strains share PR1 and PR3 whereas all the other PR islands are strain-specific. PR1 in Lpa and Lpc are almost syntenic and could be considered an ancient infection that took place before the split of the two lineages; a related region is also present in Lpg genome. This island contains several phage-related proteins, several transposases belonging to the IS4 family, an MviN virulence factor (lpa01685, LPC_2173, lpg1087), as well as other not well-defined proteins containing DNA cleavage or binding related domains. The MvinN-related protein is additional, but not equivalent to the constitutive one present on the chromosomes (see Additional file 4) of the five strains (lpa0385, LPC_0506, lpg2635, lpl2560, and lpp2688). MvinN has been described as an important factor of virulence in Salmonella typhimurium and Burkholderia pseudomallei [35]. Although its role in pathogenicity is still not clear, this protein is a homolog of the proposed lipid II flippase protein [36,37] that has no virulence activity.

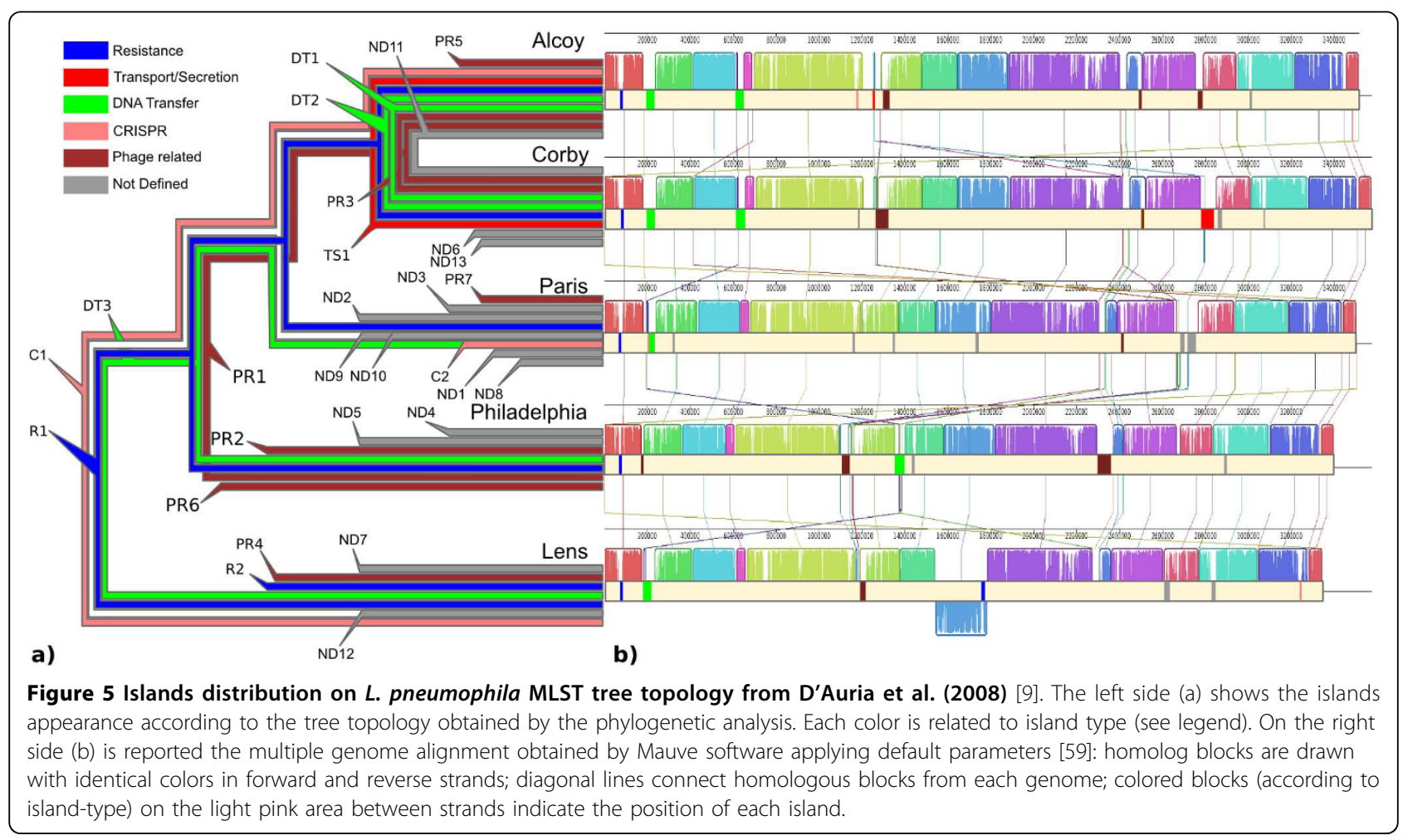


PR2 is specific to the Philadelphia strain and relies on a probable hot spot region. It contains six residual transposase-related ORFs followed by four hypothetical proteins that correspond to the beginning of the DT1ac islands.

PR3, as stated above, has been found only in the Corby and Alcoy strains. It begins with an ankyrin repeat-containing protein (LPC_1606, lpa03089). Ankyrin-domain-containing proteins have a eukaryotic origin and are related to intracellular trafficking. In Legionella ankyrin-containing proteins may be secreted by the Dot/Icm system and could play a role in intracellular bacterial replication [14,38]. The island also contains several hypothetical proteins (LPC_1607LPC_1615, lpa03090-lpa03095) and a transposase (LPC_1616, lpa03096).

PR4 is specific to the Lens strain genome and spans around $29 \mathrm{~kb}$. It seems to be a residual plasmid integrated into the chromosome, based on the presence of several proteins relating to DNA organization, such as lpl1068 containing SNF2-related protein domains that seem to be similar to helicases, and an Omp/MotB domain-containing protein (lpl1070) that may be related to structural flagella membrane proteins. Several phagerelated proteins and transposases were found, together with another predicted inner membrane protein (lpl1073). Moreover, this island contains an ORF similar to DNA damage-inducible protein J (lpl1084), and the last two ORFs (lpl1092 and lpl1093) have high homology to other plasmid maintenance killer/antidote systems. The latter has been identified in several gram-negativerelated plasmids and it is known as a regulator of bacterial programmed cellular death, although in some cases (e. g. E. coli) it is also integrated into the chromosome [39].

The Alcoy-specific island PR5 is integrated into a $[\mathrm{CAT}]-\mathrm{tRNA}^{\text {ile }}$ site. The first three ORFs code for proteins related to transposases, whereas the remainder code for hypothetical proteins with no clear function. None of these proteins appear to have any relationship with other Legionella-related ORFs, and seem to be a clear case of acquisition by HGT. Three genes with no clear function, TraK gene (lpa03390), an inner membrane protein (lpa03394), and one hypothetical protein (lpa03395), have been found to be syntenic with the Lpg and Lpl genomes (lpg2365, lpg2366, and lpg2367; lpl0071, lpl0070, and lpl006, respectively). lpa03400 is similar to a phage-related integrase. The next ORFs (on the reverse strand) are related to a cluster of genes involved in bacilysin synthesis, known as one of the simplest antibiotic peptides active against some bacteria and fungi [40] (see Additional file 5).

PR6 in the Philadelphia genome includes homologs of a type IV secretion system, mobile genetic elements, and virulence factors. It has been described in detail by Brassinga and collaborator (2003) as a $65 \mathrm{~kb}$ pathogenicity island [41].

Finally, the island PR7 in the Paris genome codes almost exclusively for hypothetical proteins. Only a putative primase/helicase (lpp2117), and a phage integrase (lpp2123) homolog seems to relate this island to phage integration with an unpredicted function.

\section{Not defined island (ND)}

Up to thirteen islands, for which it has not been possible to establish a clear role, have been identified. Interestingly, all but one (ND11 in Lpa and Lpc), are strain-specific islands. ND1, ND2, ND3, ND8, ND9, and ND10 are found in the Paris genome. ND1 hosts a complete cytochrome $o$ cluster (subunits II, I, III, IV on lpp0294, lpp0295, lpp0296, lpp0297, respectively) and a glycine/betaine transporter. ND2 contains hypothetical proteins, a CsrA (Carbon storage regulator), and is located close to the chromosomal heavy metals regulatory genes ( $\mathrm{Hel} A B C)$. ND3 is formed by two hypothetical proteins followed by the HupE/UreJ membrane protein (lpp12118), homologs of Nickel/ Cobalt type II transport systems [42]. These elements are followed by a thiocyanate hydrolase cluster for subunits gamma, alpha and beta (lpp1219, lpp1220 and lpp122, respectively) that were previously identified as unique to the Paris genome when compared to that from Lens [6]. This enzyme is the first key step in thiocyanate degradation and it is important in the detoxification processes [43]. ND8 is a small island composed mainly of transposases. Finally, for ND9 and ND10, it was not possible to propose a role. ND4 and ND5 are specific to the Philadelphia strains. ND4 is a small island containing hypothetical proteins and transposases, whereas ND5 contains a whole set of transposases and phage integrases as well as hypothetical proteins. ND6 and ND13 are specific to the Corby strain. ND6 spans a $7 \mathrm{~kb}$ region in the Corby genome with no apparently exogenous genes; it contains mainly hypothetical proteins, although some ORFs seem to be related to acetyltransferases. ND13 is syntenic with the terminal part of the Lpp island ND10 and, similarly, consists of hypothetical proteins. ND11 was found to be syntenic in both the Alcoy and Corby genomes and contains transposases and hypothetical proteins. ND7 and ND12 islands were found only in the Lens strains. ND7 is another not well-defined island consisting mostly of hypothetical proteins. It contains an ORF related to filamentation induced by a cAMP protein (lpl2288), an incomplete homolog of a HipA system, as well as the R1p island HipA/HipB system. Finally ND12 is made up exclusively of hypothetical proteins. 


\section{Conclusions}

The virulence and persistence of L. pneumophila are mainly due to specific mechanisms coded by part of its core genome that makes $L$. pneumophila able to infect, survive and replicate in macrophages [14,44-46]. Lpc has been described as one of the most virulent strains [47], while Lpp is responsible for sporadic cases but is frequently recognized worldwide [48]. Lpl was responsible for important outbreaks in France during 2003-2004 with 86 registered cases resulting in 17 deaths [6]. Finally, although the Lpg strain was the first one isolated for which the genome sequence was defined, it turned out to be not so virulent as the other [49].

Comparative genomics of five strains isolated in different parts of the world of L. pneumophila disclosed the presence of several HGT-related islands and an evident history of recombination events. Here, we reported a number of features connected to virulence that could have been exchanged, or acquired by the strains along with their evolution. The traces of these events are mainly part of the dispensable genome compartment.

The islands encountered from the dispensable pangenome compartment of the five genomes revealed factors that can give additional virulence to each strain. Alcoy and Corby strains are those in which more islands have been found related to virulence and DNA transfer activities. Multi-drug efflux systems have been found in Lpa, Lpc and Lpg, while stability systems have been found in Lpl and Lpp genomes (R1). Lpa and Lpc strains are probably potentially more resistant in the presence of heavy metals, due to an additional HelABC system in the TS1 island. Moreover, Lpa and Lpc seem to have acquired, before to their lineage split, the ability to be more successful in DNA transfer by the DT1ac and DT2ac systems. Interestingly, Alcoy strain also acquired a complete bacilysin system (PR5 island), probably by precedent phage contact after separation from the Corby lineage, which could represent an environmentally competitive advantage for this virulent strain. Lpa, Lpc the Lpg also carry an additional Mvin virulence factor, although there is no experimental evidence of its activity (island PR1). Moreover Alcoy, as well as Lens and Paris, proved to carry phage resistance systems (CRISPR on C1al and C2p islands). Several additional specific features have also been reported, although their role could not be predicted.

Finally, the data reported in this work show that the Alcoy strain possesses additional features, making it different from other previously sequenced genomes, even with the most closely related Corby strain. This finding could be related to the recurrent and sometimes mortal outbreaks recorded in the Spanish town of Alcoy.

\section{Methods}

Strains used in this work

L. pneumophila strain Alcoy 2300/99 was isolated from sputum of a patient with Legionnaires' disease (LD) and associated with the LD outbreak detected in Alcoy (Spain) in 1999. It belongs to the most predominant serogroup 1 [8]. The same strain was further isolated in other successive LD outbreaks in 2000 and 2002. The publicly available genomes of $L$. pneumophila used for comparison were retrieved from GenBank database http://www.ncbi.nlm.nih.gov/Genbank/index.html. Abbreviations and accession numbers are reported in Table 1.

\section{DNA extraction, shotgun clone libraries and sequencing}

DNA from L. pneumophila Alcoy was extracted as described in D'Auria et al. (2008) [9]. Cloning and sequencing were carried out as follows: two libraries (inserts of 1-2 and 2-10 Kb) were generated by sonication of genomic DNA, followed by cloning of the fragments using the TOPO XL PCR Cloning Kit (Invitrogen, \#K4700-10). Plasmid DNA purification was done with a Montage Plasmid Miniprep96 kit (Millipore, \#LSKP09624) on a MULTIPROBE II-Robot Liquid Handling System (Packard Bioscience). Sequencing reactions were mainly performed using the ABI PRISM BigDye Terminator v3.0 Ready Reactions Kit and resolved using the $3730 \mathrm{Xl}$ Genetic Analyzer (Applied Biosystems). To complete the assembly we used 454 pyrosequencing (Roche) performed on one half of a GS-FLX PicoTiter plate, obtaining a total of $52 \mathrm{Mb}$. The combination of both sequencing methods allowed the genome to be defined in 4 contigs. Finally, inverse PCR was employed to fill the remaining gaps and close the genome.

\section{Genome assembly and annotation}

Base-calling of each Sanger read was carried out with the "Pregap4" interface from Staden Package [50]. All reads were then checked manually by the "Trev" program and the assembly of Sanger sequences was performed by Cap4 program, both from the Staden Package [50]. The 454 reads were assembled by the Newbler assembler http://www.roche-applied-science. com and then integrated with the previous Sanger assembly. Open reading frame predictions were carried out with the Glimmer3 program [51] assigning the "lpa" locus tag to each sequence. All CDSs were searched by BLAST [52] searches against the non-redundant GenBank database, the Cluster of Orthologous Groups [53] and the Kyoto Encyclopedia of Genes and Genomes [54]. Annotation was then improved by homology searches against previously sequenced genomes of the 
Philadelphia, Lens, Paris and Corby strains (see Table 1 for genome accession numbers). Ribosomal genes were identified by BLAST searches against "nt" databases. tRNAs were identified by the tRNAscanSE software [55]. tRNA genes with anticodon CAT (tRNAIle, tRNAMet and tRNAfMet) were identified by the method described by Silva and collaborators [56].

\section{Comparative analyses}

CDSs from each genome were considered orthologous when reciprocal BLAST best hits gave at least $70 \%$ of overlap with a minimum of $80 \%$ similarity. A catalogue of orthologs was compiled. GenomeViz2 software was employed to draw genome plots [57]. Several Perl scripts were compiled in our laboratory for massive data handling (available upon request).

To define the coverage of the L. pneumophila pangenome, rarefaction curves were calculated from pools of CDS from each genome. In ecology, rarefaction is a technique applied in order to standardize and compare species richness computed from samples of different size [58]. Here, it is applied to compare gene cluster richness among multiple genomes from the same species. The L. pneumophila pangenome was then compared with pangenomes from strains belonging to $E$. coli (8 genomes), Streptococcus pyogenes (8 genomes), Staphylococcus aureus (9 genomes), Streptococcus agalactiae (8 genomes)(accession numbers are reported in Additional file 6). For each genome BLASTCLUST software was used to define gene clusters (70\% similarity and $70 \%$ overlap). Gene abundance within each cluster was used to calculate rarefaction curves by the RarefactWin.exe http://www.uga.edu/ strata/software/Software. html program.

A comparative analysis among the five strains has been carried out using the Mauve, multiple genome alignment software [59].

\section{Pangenome distribution}

All CDSs from each genome were pooled together and clustered by CD-HIT-EST software with at least $70 \%$ of overlapping and a minimum of $80 \%$ similarity [60]. One gene from each cluster was characterized by RPSBLAST best match ( $e$-values lower than $\left.10^{-15}\right)$ against the COG database (Cluster of Orthologous Groups, [53]).

\section{Determination of specific islands}

Discontinuity of the homology (synteny) between CDSs from a given genome and its ortholog in every comparison were considered to define an island. Generally, islands were defined when more than 5 consecutives CDSs were found to be specific for one strain. Syntenic Alcoy/Corby orthologous genes which did not match in the other three genomes were also considered islands. Islands were named according to their proposed function. A lowercase letter was added to the end of the name referring to the genome to which it belonged and letters were chosen according to the official locus tag definition ("a" for Alcoy, "c" for Corby, "g" for Philadelphia, "l" for Lens and "p" for Paris; e.g. "TS1a": Transport/Secretion island number 1 from the Alcoy genome). Due to the fact that the original annotations of Lpc, Lpg, Lpl and Lpp genomes often report CDSs as "hypothetical protein", similarity searches of genes within these islands were carried out against an updated Refseq (GenBank) database http://www.ncbi.nlm.nih. gov/RefSeq/.

Additional file 1: Presence/absence of islands among the five considered genomes. The presence or absence of a given island (column headers) is reported for every genome (line). Orange island is common to all strain, blue islands are strain-specific islands, red islands are common to Lpa and Lpc genomes, the green island is present in Lpp, Lpg and Lpl but not in Lpa or Lpc genomes. For the other is not possible to make a clear hypothesis about their origin.

Additional file 2: CRISPR associated gene trees. Rooted trees obtained by neighbor joining method applying Kimura distance. In bold the Legionella pneumophila str. Alcoy sequence. Relative sequences represent best hits from GenBank protein refseq database.

Additional file 3: CRISPR repeats structure in Alcoy genome. Rooted trees obtained by neighbor joining method applying Kimura distance. In bold the Legionella pneumophila str. Alcoy sequence. Relative sequences represent best hits from GenBank protein Refseq database.

Additional file 4: Mvin gene trees. Rooted trees obtained by neighbor joining method applying Kimura distance. In bold the Legionella pneumophila str. Alcoy sequence. Relative sequences represent best hits from GenBank protein Refseq database.

Additional file 5: Bacilysin containing island from Alcoy genome. Bacilysin cluster begins with an ORF homolog of a phospho-2-dehydro-3deoxyheptonate aldolase, which is an intermediate of the synthesis of chorismate (Ipa03410); the next four ORFs are homologs of bacilysin biosynthesis BacA (Ipa03409), BacB (Ipa03408), BacC (Ipa03407) and BacC (Ipa03406); Ipa03405 is a transporter of the multidrug/metabolite exporter family; Ipa0304 is a purine metabolism-related protein. Ipa03404 and Ipa3403 are related to chorismate mutase and the subsequent amino-transferase could be related to the final steps in bacilysin biosynthesis [61]. Located further along the island, Ipa03412 is a homolog of an S24-like peptidase, followed by two hypothetical proteins and a bacteriocin adenylyltransferase (Ipa03416). The PR5 island ends with an IS10-related transposase, Ipa03419, two hypothetical proteins and a phage related integrase (Ipa03425). This island demonstrates evidence of cluster acting in bacilysin-like bacteriocin production that is specific to the Alcoy genome.

Additional file 6: Genomes used for rarefaction analysis. For each organisms strain names and GenBank accession numbers are reported.

\section{Acknowledgements}

This work has been funded by contract with Consellería de Sanidad of Valencian Government to AL and AM, and by grants BFU2009-12895-CO2-01 and SAF2009-13032-CO2-01 from Ministerio de Ciencia e Innovación (MICINN) to AL and AM, respectively. NJH is recipient of a fellowship from Carlos III and GD has a research contract from CIBERESP. Sanger sequencing was carried out using facilities of the SCSIE from University of Valencia. 


\section{Author details}

${ }^{1}$ CIBER en Epidemiología y Salud Pública (CIBERESP), Barcelona, Spain. ${ }^{2}$ Area de Genómica y Salud, Centro Superior de Investigación en Salud Pública (CSISP), (Avda. de Cataluña, 21), Valencia, (46020), Spain. ${ }^{3}$ Instituto Cavanilles de Biodiversidad y Biología Evolutiva, Universitat de València, (Poligono La Coma s/n), Paterna, Valencia, (46071), Spain.

\section{Authors' contributions}

GD participated in conception, genome assembly, comparative analysis, and drafted the manuscript. NJH participated in sequencing and genome assembly FPB participated in sequencing and genome assembly and annotation. AM participated in the conception and design of the study and critically revised the manuscript. AL participated in the conception, design and discussion of the study and critically revised the manuscript. All authors have read and approved the final manuscript.

Received: 11 September 2009 Accepted: 17 March 2010

Published: 17 March 2010

\section{References}

1. Fields BS, Benson RF, Besser RE: Legionella and Legionnaires' disease: 25 years of investigation. Clin Microbiol Rev 2002, 15:506-526.

2. Lu H, Clarke M: Dynamic properties of Legionella-containing phagosomes in Dictyostelium amoebae. Cell Microbiol 2005, 7:995-1007.

3. Sabria M, Alvarez J, Dominguez A, Pedrol A, Sauca G, Salleras L, Lopez A, Garcia-Nunez MA, Parron I, Barrufet MP: A community outbreak of Legionnaires' disease: evidence of a cooling tower as the source. Clin Microbiol Infect 2006, 12:642-647.

4. Fernandez JA, Lopez P, Orozco D, Merino J: Clinical study of an outbreak of Legionnaire's disease in Alcoy, Southeastern Spain. Eur J Clin Microbiol Infect Dis 2002, 21:729-735.

5. Chien M, Morozova I, Shi S, Sheng H, Chen J, Gomez SM, Asamani G, Hill K, Nuara J, Feder $M$, et al: The genomic sequence of the accidental pathogen Legionella pneumophila. Science 2004, 305:1966-1968.

6. Cazalet C, Rusniok C, Bruggemann H, Zidane N, Magnier A, Ma L, Tichit M, Jarraud S, Bouchier C, Vandenesch F, et al: Evidence in the Legionella pneumophila genome for exploitation of host cell functions and high genome plasticity. Nat Genet 2004, 36:1165-1173.

7. Glockner G, Albert-Weissenberger C, Weinmann E, Jacobi S, Schunder E, Steinert M, Hacker J, Heuner K: Identification and characterization of a new conjugation/type IVA secretion system (trb/tra) of Legionella pneumophila Corby localized on two mobile genomic islands. Int J Med Microbiol 2008, 298:411-428.

8. Yu VL, Plouffe JF, Pastoris MC, Stout JE, Schousboe M, Widmer A, Summersgill J, File T, Heath CM, Paterson DL, Chereshsky A: Distribution of Legionella species and serogroups isolated by culture in patients with sporadic community-acquired legionellosis: an international collaborative survey. J Infect Dis 2002, 186:127-128.

9. D'Auria G, Jimenez N, Peris-Bondia F, Pelaz C, Latorre A, Moya A: Virulence factor rtx in Legionella pneumophila, evidence suggesting it is a modular multifunctional protein. BMC Genomics 2008, 9:14.

10. Horwitz MA: Phagocytosis of the Legionnaires' disease bacterium (Legionella pneumophila) occurs by a novel mechanism: engulfment within a pseudopod coil. Cell 1984, 36:27-33.

11. Tachado SD, Samrakandi MM, Cirillo JD: Non-opsonic phagocytosis of Legionella pneumophila by macrophages is mediated by phosphatidylinositol 3-kinase. PLoS One 2008, 3:e3324.

12. Ensminger AW, Isberg RR: Legionella pneumophila Dot/lcm translocated substrates: a sum of parts. Curr Opin Microbiol 2009, 12:67-73.

13. Cirillo SL, Yan L, Littman M, Samrakandi MM, Cirillo JD: Role of the Legionella pneumophila rtxA gene in amoebae. Microbiology 2002, 148:1667-1677.

14. Gomez-Valero L, Rusniok C, Buchrieser C: Legionella pneumophila: population genetics, phylogeny and genomics. Infect Genet Evol 2009, 9:727-739.

15. Medini D, Donati C, Tettelin H, Masignani V, Rappuoli R: The microbial pan-genome. Curr Opin Genet Dev 2005, 15:589-594.

16. Rocha EP: Evolutionary patterns in prokaryotic genomes. Curr Opin Microbiol 2008, 11:454-460.

17. Rasko DA, Rosovitz MJ, Myers GS, Mongodin EF, Fricke WF, Gajer P, Crabtree J, Sebaihia M, Thomson NR, Chaudhuri R, et al: The pangenome structure of Escherichia coli: comparative genomic analysis of E. coli commensal and pathogenic isolates. J Bacteriol 2008, 190:6881-6893.

18. Tettelin $\mathrm{H}$, Masignani $\mathrm{V}$, Cieslewicz MJ, Donati C, Medini D, Ward NL, Angiuoli SV, Crabtree J, Jones AL, Durkin AS, et al: Genome analysis of multiple pathogenic isolates of Streptococcus agalactiae: implications for the microbial "pan-genome". Proc Natl Acad Sci USA 2005, 102:13950-13955.

19. Lefebure T, Stanhope MJ: Evolution of the core and pan-genome of Streptococcus: positive selection, recombination, and genome composition. Genome Biol 2007, 8:R71.

20. Coscolla M, Gonzalez-Candelas F: Comparison of clinical and environmental samples of Legionella pneumophila at the nucleotide sequence level. Infect Genet Evol 2009, 9:882-888.

21. Harrison TG, Afshar B, Doshi N, Fry NK, Lee JV: Distribution of Legionella pneumophila serogroups, monoclonal antibody subgroups and DNA sequence types in recent clinical and environmental isolates from England and Wales (2000-2008). Eur J Clin Microbiol Infect Dis 2009, 28:781-791.

22. Correia FF, D'Onofrio A, Rejtar T, Li L, Karger BL, Makarova K, Koonin EV, Lewis K: Kinase activity of overexpressed HipA is required for growth arrest and multidrug tolerance in Escherichia coli. J Bacteriol 2006 188:8360-8367.

23. Korch SB, Hill TM: Ectopic overexpression of wild-type and mutant hipA genes in Escherichia coli: effects on macromolecular synthesis and persister formation. J Bacteriol 2006, 188:3826-3836.

24. Mazzariol A, Cornaglia G, Nikaido H: Contributions of the AmpC betalactamase and the AcrAB multidrug efflux system in intrinsic resistance of Escherichia coli K-12 to beta-lactams. Antimicrob Agents Chemother 2000, 44:1387-1390.

25. Hayes F: A family of stability determinants in pathogenic bacteria. $J$ Bacteriol 1998, 180:6415-6418.

26. Jiang $Y$, Yang F, Zhang $X$, Yang J, Chen L, Yan Y, Nie H, Xiong Z, Wang J, Dong J, et al: The complete sequence and analysis of the large virulence plasmid pSS of Shigella sonnei. Plasmid 2005, 54:149-159.

27. McClain MS, Hurley MC, Brieland JK, Engleberg NC: The Legionella pneumophila hel locus encodes intracellularly induced homologs of heavy-metal ion transporters of Alcaligenes spp. Infect Immun 1996, 64:1532-1540.

28. Busenlehner LS, Pennella MA, Giedroc DP: The SmtB/ArsR family of metalloregulatory transcriptional repressors: Structural insights into prokaryotic metal resistance. FEMS Microbiol Rev 2003, 27:131-143.

29. Doleans-Jordheim A, Akermi M, Ginevra C, Cazalet C, Kay E, Schneider D, Buchrieser C, Atlan D, Vandenesch F, Etienne J, Jarraud S: Growth-phasedependent mobility of the Ivh-encoding region in Legionella pneumophila strain Paris. Microbiology 2006, 152:3561-3568.

30. Samrakandi MM, Cirillo SL, Ridenour DA, Bermudez LE, Cirillo JD: Genetic and phenotypic differences between Legionella pneumophila strains. J Clin Microbiol 2002, 40:1352-1362.

31. Segal G, Russo JJ, Shuman HA: Relationships between a new type IV secretion system and the icm/dot virulence system of Legionella pneumophila. Mol Microbiol 1999, 34:799-809.

32. Molofsky AB, Swanson MS: Legionella pneumophila CsrA is a pivotal repressor of transmission traits and activator of replication. Mol Microbiol 2003, 50:445-461.

33. Barrangou R, Fremaux C, Deveau H, Richards M, Boyaval P, Moineau S, Romero DA, Horvath P: CRISPR provides acquired resistance against viruses in prokaryotes. Science 2007, 315:1709-1712.

34. Grissa I, Vergnaud G, Pourcel C: The CRISPRdb database and tools to display CRISPRs and to generate dictionaries of spacers and repeats. BMC Bioinformatics 2007, 8:172.

35. Ling JM, Moore RA, Surette MG, Woods DE: The mviN homolog in Burkholderia pseudomallei is essential for viability and virulence. Can J Microbiol 2006, 52:831-842.

36. Inoue A, Murata Y, Takahashi H, Tsuji N, Fujisaki S, Kato J: Involvement of an essential gene, mviN, in murein synthesis in Escherichia coli. $J$ Bacteriol 2008, 190:7298-7301

37. Ruiz N: Bioinformatics identification of MurJ (MviN) as the peptidoglycan lipid II flippase in Escherichia coli. Proc Natl Acad Sci USA 2008, 105:15553-15557.

38. Al-Khodor S, Price CT, Habyarimana F, Kalia A, Abu Kwaik Y: A Dot/lcmtranslocated ankyrin protein of Legionella pneumophila is required for 
intracellular proliferation within human macrophages and protozoa. Mol Microbiol 2008, 70:908-923.

39. Jensen RB, Gerdes K: Programmed cell death in bacteria: proteic plasmid stabilization systems. Mol Microbiol 1995, 17:205-210.

40. Kenig M, Abraham EP: Antimicrobial activities and antagonists of bacilysin and anticapsin. J Gen Microbiol 1976, 94:37-45.

41. Brassinga AK, Hiltz MF, Sisson GR, Morash MG, Hill N, Garduno E, Edelstein PH, Garduno RA, Hoffman PS: A 65-kilobase pathogenicity island is unique to Philadelphia-1 strains of Legionella pneumophila. J Bacteriol 2003, 185:4630-4637.

42. Rodionov DA, Hebbeln P, Gelfand MS, Eitinger T: Comparative and functional genomic analysis of prokaryotic nickel and cobalt uptake transporters: evidence for a novel group of ATP-binding cassette transporters. J Bacteriol 2006, 188:317-327.

43. Bezsudnova EY, Sorokin DY, Tikhonova TV, Popov VO: Thiocyanate hydrolase, the primary enzyme initiating thiocyanate degradation in the novel obligately chemolithoautotrophic halophilic sulfur-oxidizing bacterium Thiohalophilus thiocyanoxidans. Biochim Biophys Acta 2007, 1774:1563-1570.

44. Bandyopadhyay P, Liu S, Gabbai CB, Venitelli Z, Steinman HM: Environmental mimics and the Lvh type IVA secretion system contribute to virulence-related phenotypes of Legionella pneumophila. Infect Immun 2007, 75:723-735.

45. Franco IS, Shuman HA, Charpentier $X$ : The perplexing functions and surprising origins of Legionella pneumophila type IV secretion effectors. Cell Microbiol 2009, 11(10):1435-43.

46. Skriwan C, Fajardo M, Hagele S, Horn M, Wagner M, Michel R, Krohne G, Schleicher M, Hacker J, Steinert M: Various bacterial pathogens and symbionts infect the amoeba Dictyostelium discoideum. Int J Med Microbiol 2002, 291:615-624.

47. Shevchuk O, Batzilla C, Hagele S, Kusch H, Engelmann S, Hecker M, Haas A, Heuner K, Glockner G, Steinert M: Proteomic analysis of Legionellacontaining phagosomes isolated from Dictyostelium. Int J Med Microbiol 2009, 299(7):489-508.

48. Cazalet C, Jarraud S, Ghavi-Helm Y, Kunst F, Glaser P, Etienne J, Buchrieser C: Multigenome analysis identifies a worldwide distributed epidemic Legionella pneumophila clone that emerged within a highly diverse species. Genome Res 2008, 18:431-441.

49. Jepras Rl, Fitzgeorge RB, Baskerville A: A comparison of virulence of two strains of Legionella pneumophila based on experimental aerosol infection of guinea-pigs. J Hyg (Lond) 1985, 95:29-38.

50. Staden R: The Staden sequence analysis package. Mol Biotechnol 1996, 5:233-241.

51. Delcher AL, Harmon D, Kasif S, White O, Salzberg SL: Improved microbial gene identification with GLIMMER. Nucleic Acids Res 1999, 27:4636-4641.

52. Altschul SF, Gish W, Miller W, Myers EW, Lipman DJ: Basic local alignment search tool. J Mol Biol 1990, 215:403-410.

53. Tatusov RL, Fedorova ND, Jackson JD, Jacobs AR, Kiryutin B, Koonin EV, Krylov DM, Mazumder R, Mekhedov SL, Nikolskaya AN, et al: The COG database: an updated version includes eukaryotes. BMC Bioinformatics 2003, 4:41.

54. Moriya Y, Itoh M, Okuda S, Yoshizawa AC, Kanehisa M: KAAS: an automatic genome annotation and pathway reconstruction server. Nucleic Acids Res 2007, 35:W182-185.

55. Lowe TM, Eddy SR: tRNAscan-SE: a program for improved detection of transfer RNA genes in genomic sequence. Nucleic Acids Res 1997, 25:955-964.

56. Silva FJ, Belda E, Talens SE: Differential annotation of tRNA genes with anticodon CAT in bacterial genomes. Nucleic Acids Res 2006, 34:6015-6022.

57. Ghai R, Hain T, Chakraborty T: GenomeViz: visualizing microbial genomes. BMC Bioinformatics 2004, 5:198.

58. Sanders HL: Marine Benthic Diversity: A Comparative Study. The American Naturalist 1968, 102:243-282.

59. Darling AC, Mau B, Blattner FR, Perna NT: Mauve: multiple alignment of conserved genomic sequence with rearrangements. Genome Res 2004, 14:1394-1403.

60. Li W, Godzik A: Cd-hit: a fast program for clustering and comparing large sets of protein or nucleotide sequences. Bioinformatics 2006, 22:1658-1659.
61. Hilton MD, Alaeddinoglu NG, Demain AL: Synthesis of bacilysin by Bacillus subtilis branches from prephenate of the aromatic amino acid pathway. J Bacteriol 1988, 170:482-484.

doi:10.1186/1471-2164-11-181

Cite this article as: D'Auria et al: Legionella pneumophila pangenome reveals strain-specific virulence factors. BMC Genomics 2010 11:181.

\section{Submit your next manuscript to BioMed Central and take full advantage of:}

- Convenient online submission

- Thorough peer review

- No space constraints or color figure charges

- Immediate publication on acceptance

- Inclusion in PubMed, CAS, Scopus and Google Scholar

- Research which is freely available for redistribution

Submit your manuscript at www.biomedcentral.com/submit
Biomed Central 